\title{
Cavernous sinus thrombosis secondary to sinusitis: a rare and life-threatening complication
}

\author{
Cam-Tu E. Nguyen • Ricardo Faingold
}

Received: 29 September 2008 /Revised: 15 November 2008 / Accepted: 9 December 2008 / Published online: 27 January 2009

(C) Springer-Verlag 2009

A 17-year-old girl presented with headache, and right third and left sixth nerve palsies. MRI was performed. Coronal T1-W image after gadolinium administration (Fig. 1) demonstrated enlargement and pathological enhancement of the left cavernous sinus (arrow) with narrowing of the left internal carotid artery (arrowhead). Axial T2-W image (Fig. 2) showed opacification with high signal intensity in the ethmoid air cells. There was sphenoid sinus signal abnormality within an enlarged left cavernous sinus (arrow) involving a narrowed carotid artery (arrowhead).

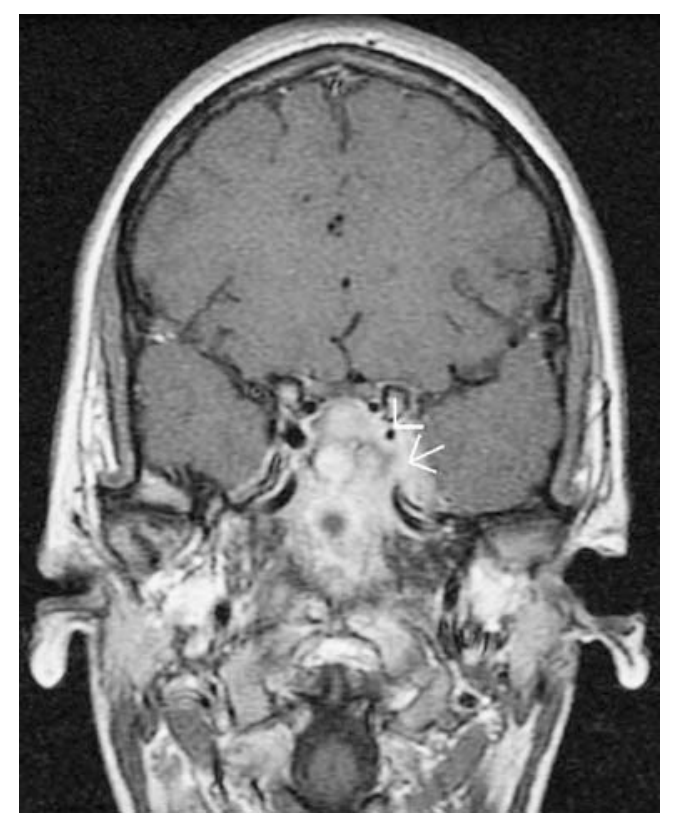

Fig. 1 Coronal T1-W image after gadolinium administration

C.-T. E. Nguyen $\cdot$ R. Faingold $(\bowtie)$

Department of Medical Imaging,

The Montreal Children's Hospital, McGill University,

2300 Tupper St.,

Montreal, Canada H3H 1P3

e-mail: ricardo.faingold@muhc.mcgill.ca

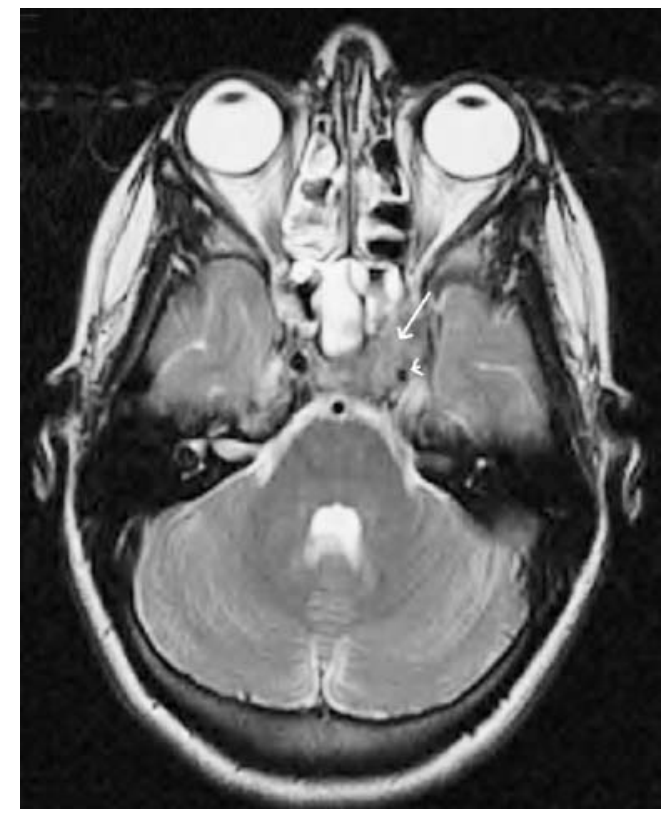

Fig. 2 Axial T2-W image

Cavernous sinus thrombosis/thrombophlebitis (CST) is a rare, albeit potentially life-threatening, complication of paranasal sinusitis [1]. CST is most often associated with sphenoid or ethmoid disease and is spread through afferent and efferent venous extension via a network of valveless veins and also direct extension [2]. Signs and symptoms include fever, headache, ptosis, proptosis, chemosis, external ophthalmoplegia and decreased corneal reflex [1]. A high index of suspicion and emergent imaging are crucial to making an early and accurate diagnosis.

\section{References}

1. Ebright JR, Pace MT, Niazi AF (2001) Septic thrombosis of the cavernous sinuses. Arch Intern Med 161:2671-2676

2. Gallagher RM, Gross CW, Phillips CD (1998) Suppurative intracranial complications of sinusitis. Laryngoscope 108:1635-1642 